\title{
TAXONOMIA POLIFÁSICA PARA IDENTIFICAÇÃO DE $A$ SPERGILLUS SEÇÃO FLAVI: UMA REVISÃO
}

Fernanda Chaves da Silva ${ }^{1}$, Sara Maria Chalfoun ${ }^{2}$, Luis Roberto Batista ${ }^{3}$, Cledir Santos ${ }^{4}$, Nelson Lima 5 .

Resumo: O gênero Aspergillus é um dos principais grupos de micro-organismos em importância para a indústria, a agricultura e a saúde pública. A mais importante publicação para a identificação e a classificação desse gênero foi baseada em critérios fenotípicos. Entretanto, devido à variabilidade das características fenotípicas, reinvestigações têm sido realizadas. Dessa forma, este trabalho tem como objetivo apresentar as principais técnicas utilizadas para identificar e classificar espécies do gênero Aspergillus seção Flavi. Nesse sentido, nossa abordagem integrará a Taxonomia Polifásica pautada nas características micro e macromorfológicas, fisiológicas, na produção de metabólitos, dados moleculares e no uso da espectrometria de massas pela técnica de MALDI-TOF MS.

Palavras-chave: Aspergillus; Abordagem polifásica; MALDI-TOF MS.

\section{POLYPHASIC TAXONOMY FOR IDENTIFICATION OF ASPERGILLUS SECTION FLAVI: A REVIEW}

Abstract: The Aspergillus genre is one of the main groups of microorganisms in terms of relevance to industry, agriculture, and public health. The most important publication concerning

1 Programa de Pós-Graduação em Microbiologia Agrícola, Universidade Federal de Lavras, Lavras (MG), Brasil; Instituto Federal do Espírito Santo, Campus Barra de São Francisco, Barra de São Francisco (ES), Brasil; CEB - Centro de Engenharia Biológica, Micoteca da Universidade do Minho, Universidade do Minho, Braga, Portugal.E-mail para contato: fernanda.silva@ifes.edu.br.

2 Empresa de Pesquisa Agropecuária de Minas Gerais (EPAMIG), Universidade Federal de Lavras, Lavras (MG), Brasil.

3 Departamento de Ciência dos Alimentos, Universidade Federal de Lavras, Lavras (MG), Brasil.

4 Programa de Pós-Graduação em Microbiologia Agrícola, Universidade Federal de Lavras, Lavras (MG), Brasil; CEB - Centro de Engenharia Biológica, Micoteca da Universidade do Minho, Universidade do Minho, Braga, Portugal.

5 Programa de Pós-Graduação em Microbiologia Agrícola, Universidade Federal de Lavras, Lavras (MG), Brasil; CEB - Centro de Engenharia Biológica, Micoteca da Universidade do Minho, Universidade do Minho, Braga, Portugal. 
this genre identification and classification was based in fenotipic criteria. However, due to the variability of the fenotipic characteristics, new investigations have been made. This work, thus, aims to present the main technics used to identify and classify species of the Aspergillus genre section Flavi. So, our approach will contemplate the Polyphasic Taxonomy based on micro-, macromorphological, and physiological characteristics, as well as on the production of metabolits, molecular data, and on the use of mass spectrometry through the MALDI-TOF MS technic.

Keywords: Aspergillus; Polyphasic approach; MALDI-TOF MS. 


\section{INTRODUÇÃO}

Os fungos são organismos economicamente importantes, amplamente distribuídos na natureza. São empregados na produção de alimentos, fármacos, enzimas e ácidos orgânicos (PATERSON, 2008). Entretanto, existem fungos na natureza considerados patógenos de plantas e deterioradores de alimentos. Os problemas causados pelo desenvolvimento desses últimos em alimentos e em suas matérias-primas são motivos de preocupação para a indústria de alimentos e para a saúde pública. Não apenas pelo fato de reduzir consideravelmente o valor nutritivo dos alimentos, mas também pela produção de metabólitos secundários tóxicos denominados micotoxinas, que podem provocar intoxicações em seres humanos e animais (RODRIGUES et al., 2009).

Dentre as micotoxinas encontradas em alimentos, as aflatoxinas são as mais importantes. Tais substâncias são sintetizadas por várias espécies do gênero Aspergillus, mantendo-se como representantes mais importantes os fungos Aspergillus flavus, produtores das aflatoxinas do grupo B, e Aspergillus nomius e Aspergillus parasiticus, produtores das aflatoxinas dos grupos B e G (KLICH, 2007). As aflatoxinas são conhecidas por serem compostos mutagênicos, carcinogênicos e teratogênicos. A exposição por ingestão de aflatoxinas pode levar ao desenvolvimento de sérias condições clínicas que variam consideravelmente dependendo da espécie animal, dose, estado nutricional, idade e gênero (HUSSEIN; BRASEL, 2001).

Atualmente, a identificação e classificação de Aspergillus seção Flavi baseiamse, principalmente, nas características morfológicas, no perfil bioquímico e molecular (BATISTA et al., 2008; RAPER; FENNEL, 1965; SAMSON et al., 2000). Embora estas espécies apresentem diferenças fisiológicas, as características morfológicas e mesmo genéticas são difíceis de diferenciar. Neste sentido, a taxonomia deste grupo de fungos é considerada altamente complexa e tem sido objeto de grande interesse para os pesquisadores, com o objetivo de esclarecer o conceito de espécie e limites dentro da seção. Recentemente, a análise espectral por MALDI-TOF ICMS tem sido utilizada 
como uma técnica potencial na identificação de fungos (SANTOS et al., 2010). Entretanto, nenhum dos métodos isolados tem sido aceito como ideal para identificação de micro-organismos. Como resultado, a abordagem polifásica tem sido adotada e relatada em vários estudos (FENSELAU; DEMIREV, 2001; HONG et al., 2005; RODRIGUES et al., 2011).

Dessa forma, este trabalho tem como objetivo apresentar as principais técnicas supracitadas utilizadas para identificar e classificar espécies do gênero Aspergillus seção Flavi.

\section{REVISÃO DE LITERATURA}

\section{Gênero Aspergillus seção Flavi}

Espécies do gênero Aspergillus são contaminantes de vários substratos. Em regiões tropicais e sub-tropicais, sua ocorrência é mais comum que a do genêro Penicillium. Muitas espécies têm atraído atenção como patógenos humano e animal devido a sua habilidade de produzir metabólitos tóxicos (micotoxinas). Outras espécies são importantes para aplicação na fermentação de produto alimentício oriental e para aplicação industrial na produção de ácidos orgânicos, metabólitos secundários, enzimas e na biotransformação de químicos (SAMSON; VARGA, 2010).

O gênero Aspergillus é um anamórfico pertencente à família Trichocomaceae, ordem Eurotiales, subclasse Eurotiomycetidae, classe Eurotiomycetes, filo Ascomycota. Sua reprodução é assexuada e caracteriza-se pela produção de fiálides e conídios em cadeia. O conidióforo de Aspergillus é simples, usualmente aseptado e termina numa vesícula, onde ficam inseridas as fiálides. Algumas espécies podem produzir células Hülle ou esclerócios. Muitas espécies de Aspergillus apresentam telemorfos e se reproduzem sexuadamente. (KLICH, 2002).

A taxonomia de Aspergillus é complexa e encontra-se sempre em evolução. A identificação de Aspergillus tem sido tradicionalmente baseada na caracterização 
morfológica e cultural (RAPER; FENNEL, 1965; SAMSON et al., 2000). O perfil bioquímico e os traços ultramicroscópicos, por exemplo, a ornamentação dos esporos, passaram a suplementar as características para identificação da espécie (KOZAKIEWICZ, 1989). No entanto, em várias seções, a variação morfológica deste gênero ocorre, resultando em esquemas taxonômicos discutíveis (HONG; GREENE; DORNER, 1995; SAMSON et al., 2004). Neste sentido, a taxonomia de Aspergillus e seus telemorfos recentemente têm sido reinvestigada, utilizando-se uma abordagem polifásica interagindo características bioquímicas, ecológicas, genéticas, moleculares, a fim de examinar a variabilidade dentro da espécie e adicionar ou reclassificar as espécies dentro do gênero (FRISVAD; SKOUBOE; SAMSON, 2005; HORN, 1997; ITO et al., 2001; LI et al., 1998; PATERSON, 2000; PETERSON, 2008; PETERSON et al., 2001; PILDAIN et al., 2008; RIGÓ et al., 2002). Manuais e monografias importantes dedicados à taxonomia Aspergillus são listados na Tabela 1.

Tabela 1. Importantes tratamentos taxonômicos e manuais de identificação para o gênero Aspergillus 


\begin{tabular}{|c|c|}
\hline ANO & REFERÊNCIAS \\
\hline 1926 & Thom, C., and Church, M. The Aspergilli (Baltimore: Williams \& Wilkins) \\
\hline 1954 & Thom, C., and Raper, K.B. A Manual of the Aspergilli (Baltimore: Williams \& Wilkins) \\
\hline 1965 & Raper, K.B., and Fennell, D.I. The Genus Aspergillus (Baltimore: Williams \& Wilkins) \\
\hline 1985 & $\begin{array}{l}\text { Samson, R.A., and Pitt, J.I. Advances in Penicillium and Aspergillus Systematics (New York: } \\
\text { Plenum) }\end{array}$ \\
\hline 1988 & $\begin{array}{l}\text { Klich, M.A., and Pitt, J.I. A Laboratory Guide to Common Aspergillus Species and Their } \\
\text { Teleomorphs (North Ryde, Australia: Division of Food Processing). }\end{array}$ \\
\hline 1989 & Kozakiewicz, A. Aspergillus Species on Stored Products (Wallingford: CAB International) \\
\hline 1990 & $\begin{array}{l}\text { Samson, R.A, and Pitt, J.I. eds. Modern Concepts in Penicillium and Aspergillus } \\
\text { Classification (New York: Plenum Press) }\end{array}$ \\
\hline 2000 & $\begin{array}{l}\text { Samson, R.A, and J. I. Pitt, eds. Integration of Modern Taxonomic Methods for Penicillium } \\
\text { and Aspergillus classification (Amsterdam: Harwood Academic Publications) }\end{array}$ \\
\hline 2002 & $\begin{array}{l}\text { Klich, M.A. Identification of Common Aspergillus Species (Utrecht: Centraalbureau voor } \\
\text { Schimmelcultures). }\end{array}$ \\
\hline 2008 & $\begin{array}{l}\text { Samson, R.A., and Varga, J. Aspergillus systematics in the genomic era (Utrecht: CBS } \\
\text { Fungal Biodiversity Centre) }\end{array}$ \\
\hline
\end{tabular}

Considerando a maioria dos esquemas tradicionais de identificação, Aspergillus seção Flavi inclui seis espécies economicamente importantes: Aspergillus flavus, Aspergillus parasiticus, Aspergillus nomius, Aspergillus oryzae, Aspergillus sojae e Aspergillus tamarii (Figura 1). Apesar de essas espécies serem intimamente relacionadas morfologica e filogeneticamente, dividem-se em dois grupos, pela capacidade de produção de aflatoxinas (GODET; MUNAUT, 2010). O primeiro grupo inclui $A$. flavus, $A$. parasiticus e $A$. nomius, espécies que são produtoras de aflatoxinas, consideradas um grande problema para saúde humana e animal, e o segundo grupo compreende os isolados chamados de espécies domesticadas, A. oryzae, A. sojae e $A$. tamarii, utilizados no processo de fermentação de alimentos em alguns países asiáticos e aplicados na indústria para a produção de enzimas (HEDAYATI et al., 2007; KUMEDA; ASAO, 2001). 
Vários estudos têm mostrado evidências de que a espécie $A$. flavus pode consistir em um grupo parafilético dos isolados (GEISER et al., 2000; PILDAIN et al., 2008), e alta intra-especificidade, diversidade metabólica e genética foram frequentemente referidas para populações de A. flavus (PILDAIN et al., 2008; RODRIGUES et al., 2009). Ao lado de Aspergillus fumigatus, é a segunda mais comum causa da aspergilose invasiva e não invasiva em seres humanos e animais (DENNING et al., 2003) e, em algumas áreas geográficas, é o principal agente causador da aspergilose. Estes fungos produzem aflatoxinas, agentes naturais cancerígenos, e ácido ciclopiazônico (CPA), que é tóxico para uma variedade de animais e humanos (HEDAYATI et al., 2007; YU et al., 2005). Além disso, é um patógeno oportunista de muitas culturas (milho, algodão, amendoim) podendo contaminá-las com aflatoxinas. Como outras espécies de Aspergillus, A. flavus tem ampla distribuição geográfica, sendo encontrado especialmente em áreas cultivadas. É um fungo saprófita capaz de sobreviver em muitas fontes de nutrientes orgânicos como: árvore, madeira em decomposição, forragem animal, algodão, pilhas de composto, cadáveres de insetos e animais, grãos armazenados. Cresce melhor com atividade de água (aw) entre 0,86 e 0,96 . A temperatura ótima para seu cresccimento é de $37^{\circ} \mathrm{C}$ mas, o crescimento dos fungos pode ser observados em temperaturas que variam entre 12 a $48^{\circ} \mathrm{C}$. Tal temperatura ótima de crescimento contribui para sua patogenicidade em seres humanos (HEDAYATI et al., 2007).

Tradicionalmente, A. flavus tem sido relatado como produtor de aflatoxinas B (AFBs) e ácido ciclopiazônico (CPA), mas não aflatoxinas G (AFGs). Com base nas características dos esclerócios produzidos, os isolados $A$. flavus podem ser divididos em dois fenótipos. A estirpe tipo-S produz numerosos esclerócios pequenos (diâmetro médio, $400 \mathrm{~mm}$ ). A estirpe tipo-L produz menos esclerócios, mas estes maiores. Dentro da estirpe $\mathrm{S}$, alguns isolados, denominados $\mathrm{SB}$, produzem somente aflatoxinas $\mathrm{B}$, enquanto outros, com o nome SBG, produzem tanto aflatoxinas B quanto G (COTTY, 1989). Os isolados tipo-S têm sido referidos como atípicos, produzindo microesclerócios (SAITO; TSURATA, 1993) e A. flavus var. parvisclerotigenu 
(GEISER et al., 2000). As estirpes microesclerotiais diferem de A. flavus e, portanto, tem sido sugerido que elas representam um taxon separado de A. flavus (FRISVAD; SKOUBOE; SAMSON, 2005; GEISER et al., 2000). A filogenia molecular sugere que os isolados SB estão intimamente relacionados com a cultura do tipo A. flavus e outros isolados de estirpe tipo-L (EGEL et al., 1994).

Aspergillus parasiticus e A. flavus estão intimamente relacionados, mas são separáveis com base na sequência de DNA e em análises por AFLP (BARROS et al., 2007; PETERSON, 2008). Fenotipicamente são facilmente distintos, já que $A$. parasiticus produz aflatoxinas do grupo B e G mas não CPA (HORN et al., 1996). Klich e Pitt (1988) completam que esta diferenciação deve levar em consideração a cor da colônia e textura da parede dos conídios. Ademais, populações de A. parasiticus apresentam uma grande diversidade genética indicada pelo grande número de grupos vegetativamente compatíveis (HORN; GREENE; DORNER, 1995). Aspergillus sojae e Aspergillus toxicarius não têm sido claramente distintos de $A$. parasiticus (PILDAIN et al., 2008). Outras espécies intimamente relacionadas com A. parasiticus, também produzindo AFBs e AFGs, mas morfologicamente semelhante a A. flavus, foram recentemente descritas como Aspergillus arachidicola (PILDAIN et al., 2008).

A espécie $A$. nomius é morfologicamente semelhante a $A$. flavus, mas apresenta um perfil micotoxigênico similar ao $A$. parasiticus (KURTZMAN; HORN; HESSELTINE, 1987; PETERSON et al., 2001), tendo sido, originalmente, isolado do mofo de trigo nos EUA e, depois, de vários substratos na Índia, no Japão e na Tailândia. Recentemente, tem sido relatado como um contaminante importante e produtor de aflatoxinas na castanha do Brasil (JOHNSSON et al., 2008; OLSEN et al., 2008).

A. flavus, A. parasiticus, A. oryzae e A. sojae têm demonstrado alto grau de parentesco pelo DNA e similar tamanho do genoma. Com base na complementaridade do DNA, A. flavus e A. oryzae, assim como A. parasiticus e A. sojae, foram considerados praticamente impossíveis de discriminar, já que a similaridade do DNA foi de $100 \%$ e $91 \%$, respectivamente (KURTZMAN; HORN; HESSELTINE, 1987). A. oryzae e $A$. sojae foram considerados variantes não-toxigênicos de $A$. flavus e $A$. 
parasiticus, respectivamente. Estudos filogenéticos indicaram que $A$. oryzae pode ter-se originado de um ancestral não-aflatoxigênico de A. flavus (CHANG; EHRLICH; HUA, 2006; GEISER et al., 2000; GEISER; PITT; TAYLOR, 1998), ou que tenham perdido a capacidade de produzir AFs durante o processo de domesticação (SAMSON et al., 2000). Geiser, Pitt e Taylor (1998) sugerem que A. oryzae e A. sojae devam ser mantidos como espécies separadas por razões práticas, a fim de evitar confusão na indústria de alimentos.

No grupo das espécies de $A$. tamarii, os isolados apresentam cor castanhobronze. São tradicionalmente utilizados na indústria de alimento oriental, como não aflatoxigênicos. Goto, Wicklow e Ito (1996) relataram a produção de aflatoxinas por um isolado definido como A. tamarii, no entanto, Ito et al. (2001) descreveram mais tarde este isolado como uma nova espécie intimamente relacionada, Aspergillus pseudotamarii.

Recentemente, novas espécies têm sido descritas para esta seção. Soares et al. (2011) descrevem três novas espécies produtoras de aflatoxinas, Aspergillus mottae, Aspergillus sergii e Aspergillus transmontanensis, isolados de amêndoas portuguesas e milho. Gonçalves et al. (2012), durante uma pesquisa sobre a incidência de Aspergillus em ambientes clínicos, encontraram alguns isolados interessantes que foram morfologicamente semelhantes ao Aspergillus parasiticus, mas diferiram na cor das colônias e no padrão de sua ornamentação dos conídios, constituindo uma nova espécie, Aspergillus novoparasiticus. 


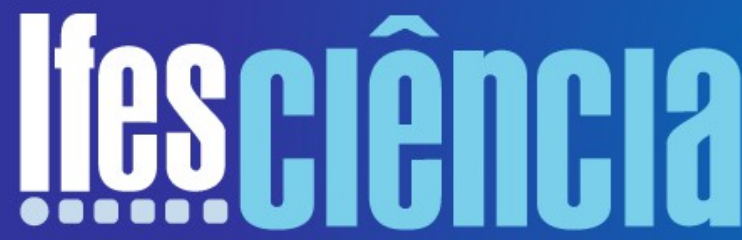

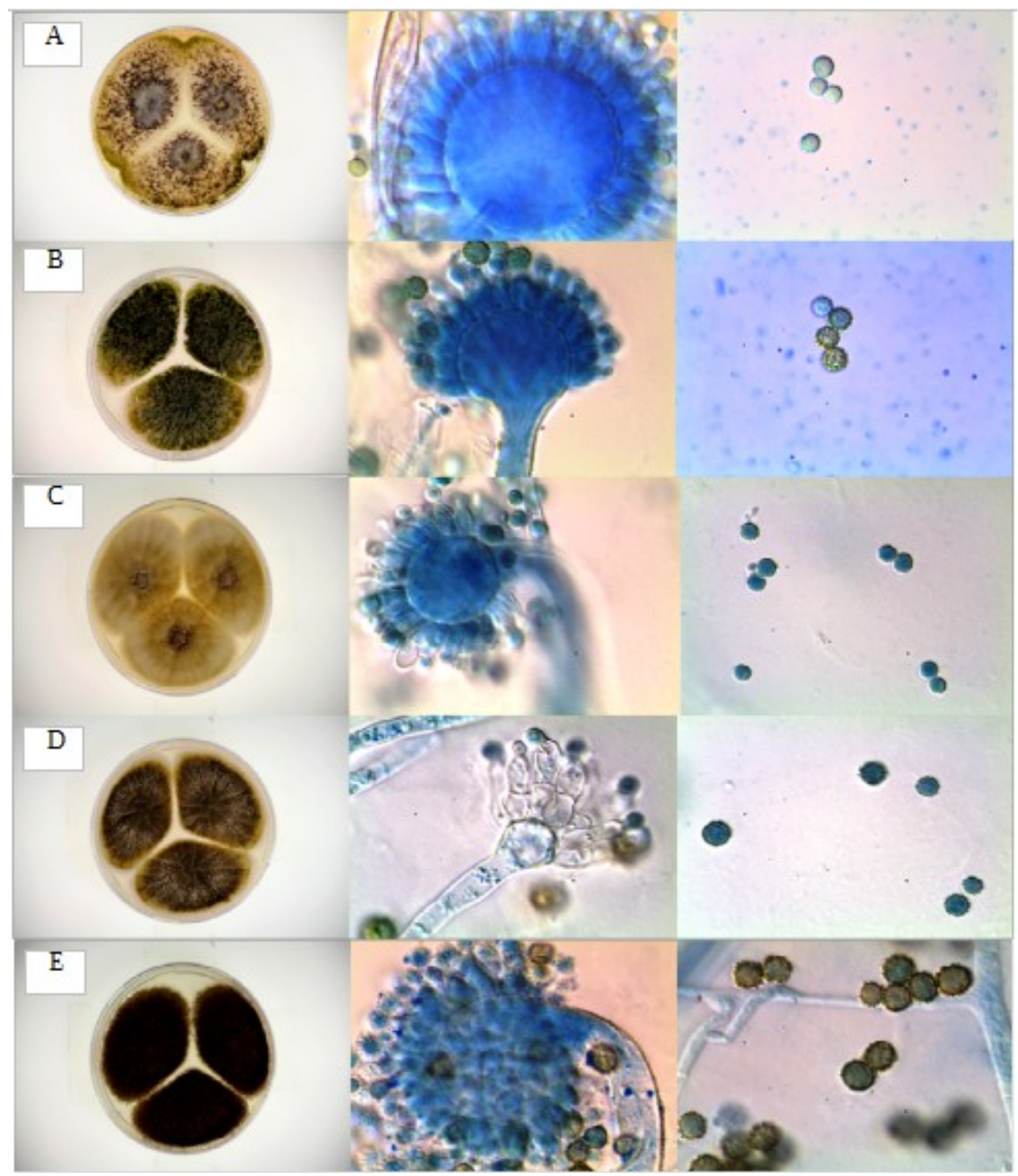

Figura 1. Macro e microfotografias das espécies Aspergillus flavus, Aspergillus parasiticus, Aspergillus oryzae, Aspergillus sojae e Aspergillus tamarii.

\section{Identificação de espécies do gênero Aspergillus - Abordagem Polifásica}

A nova taxonomia de espécies de Aspegillus é baseada em uma abordagem polifásica integrando caracteres fenotípicos, juntamente com sequências de DNA. Em um abordagem polifásica, micro e macromorfologia, fisiologia, metabólitos produzidos 


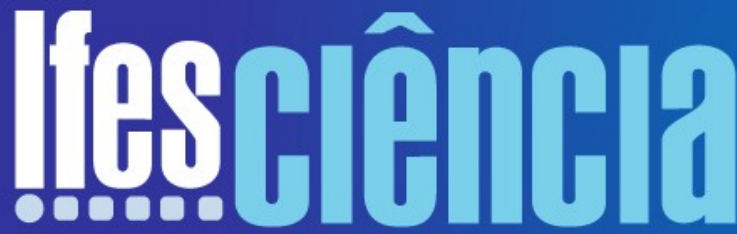

e dados moleculares são todos importantes e, em princípio, nenhum dos métodos deve ser subestimado. Em particular o perfil de extrólitos provou ser específico para táxon, e isso tem contribuído para um conceito de espécie estável. Mas dados da sequência do DNA também têm sido muito valiosos nas revisões críticas de espécies e sua taxonomia e filogenia (SAMSON; HONG; FRISVAD, 2006). Um esquema de identificação de Aspegillus com o uso da abordagem polifásica é demonstrado na Figura 2.

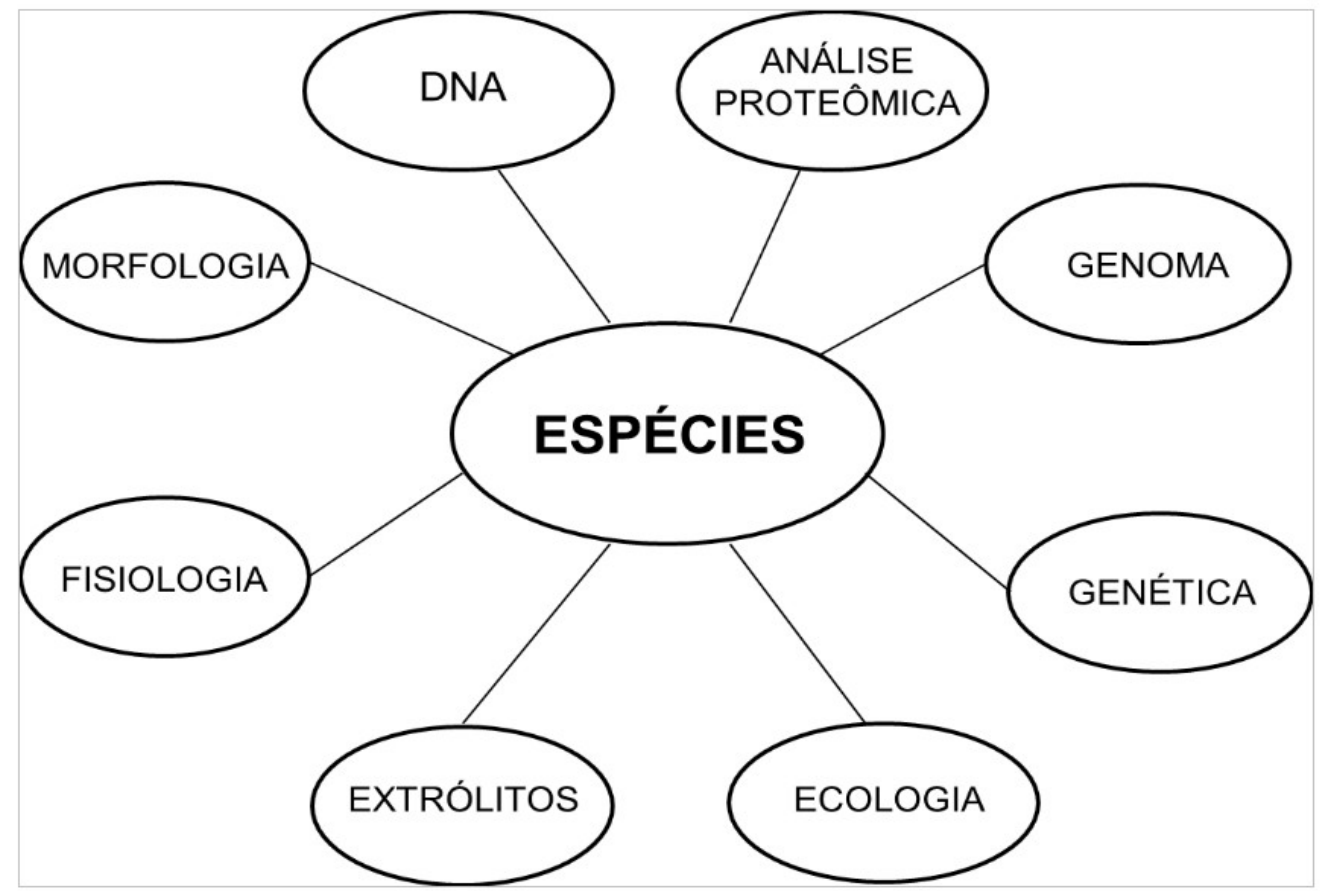

Figura 2. Esquema mostrando a integração de diferentes características que podem ser combinadas para uma classificação taxonômica polifásica de uma espécie de Aspergillus (SAMSON; HONG; FRISVAD, 2006, adaptado e atualizado).

Características morfológicas e fisiológicas

A identificação de Aspergillus tem sido tradicionalmente baseada na caracterização morfológica (RAPER; FENNEL, 1965; SAMSON et al., 2000). Características macromorfológicas incluem cor das colônias em vários meios de cultura, diâmetro da colônia, cor do reverso da colônia, produção de exsudatos e pigmentos solúveis, presença de esclerócios e cleistotécio. A caracterização micromorfológica está 
relacionada principalmente com a forma de seriação da cabeça conidial, o tamanho da vesícula, a morfologia de conídios, a presença de células Hülle e a morfologia dos esclerócios (KLICK, 2002).

Além de caracteres morfológicos, isolados de Aspergillus podem também ser caracterizados por seus perfis de metabólitos secundários, por sua taxa de crescimento em determinada temperatura e atividade de água, por seu crescimento em ágar sacarosecreatina e pela cor dos conídios (SAMSON et al., 2007). Os metabólitos secundários podem ser usados para o reconhecimento de espécies devido a sua especificidade entre espécies (FRISVAD, 1989; LARSEN et al., 2005). Praticamente todas as espécies de Aspergillus produzem uma combinação única de diferentes tipos de compostos orgânicos pequenos como policetídeos, peptídeos não-ribossomais, terpenoides, assim como muitos outros compostos de origem biossintética mista. Alguns destes compostos são mesmo exclusivos de uma única espécie. O fato de os metabólitos secundários serem bons caracteres fenotípicos para o reconhecimento de espécies é suportado por recentes estudos sobre o sequenciamento do genoma completo de importantes Aspergillus concluindo que importantes diferenças genômicas entre as espécies estão frequentemente relacionadas à produção desses metabólitos (GALAGAN et al., 2005).

$\mathrm{O}$ perfil bioquímico de Aspegillus seção Flavi está diretamente relacionado à produção de aflatoxinas. De uma forma geral, A. flavus produz aflatoxinas B (AFBs) e ácido ciclopiazônico (CPA), mas não aflatoxinas G (AFGs). A parasiticus produz a aflatoxina G1 e G2, além de B1 e B2, mas não ácido ciclopiazônico (BENNETT; KLICH, 2003; YU et al., 2005). Aflatoxinas são produzidas por algumas espécies de Aspergillus em outros complexos flavus, incluindo, A. toxicarius, A. nomius, $A$. bombycis e $A$. pseudotamarii produtor de CPA. A. oryzae tem sido muito utilizado no oriente para preparar vários tipos de produtos alimentares, uma vez que pode produzir CPA e ácido $\beta$-nitropropiônico, mas não produz a aflatoxina. Além disso, $A$. oryzae, $A$. parasiticus, A. sojae, A. nomius, A. bombycis, A. tamarii, A. caelatus e A. pseudotamarii podem produzir ácido kójico (VARGA et al., 2003). A Tabela 2 mostra a produção de aflatoxinas e alguns eletrólitos por Aspergillus seção Flavi. 
Tabela 2 - Produção de aflatoxinas e outros extólitos de espécies Aspergillus seção Flavi

\begin{tabular}{|c|c|c|c|c|c|c|c|c|}
\hline & Ácido Kojic & AFLA Tipo B & AFLA Tipo G & CPA & Ácido Aspergilico & Ocratoxina A & Chrysogine & Parasiticolide \\
\hline Aspergillus arachidicola & + & + & + & - & + & - & + & + \\
\hline Aspergillus avenaceus & - & - & - & - & - & - & - & - \\
\hline Aspergillus bombycis & + & + & + & - & $+1-$ & - & $-1+$ & - \\
\hline Aspergillus caelatus & + & - & - & + & - & - & - & - \\
\hline Aspergillus flavus & + & $+1-$ & - & + & + & - & - & - \\
\hline Aspergilus lanosus & + & - & - & - & - & - & - & - \\
\hline Aspergillus leporis & + & - & - & - & - & - & - & - \\
\hline Aspergillus minisclerotium & + & + & + & + & + & - & - & - \\
\hline Aspergillus nomius & + & + & + & - & + & - & $-1+$ & - \\
\hline $\begin{array}{l}\text { Aspergillus oryzae } \\
\text { (domesticated from } \\
\text { A. flavus) }\end{array}$ & + & - & - & + & - & - & - & - \\
\hline Aspergillus parasiticus & + & + & + & - & + & - & - & + \\
\hline Aspergillus parvisclerotigemus & + & + & + & + & + & - & - & - \\
\hline Aspergillus pseudotamarii & + & + & - & + & - & - & - & - \\
\hline $\begin{array}{l}\text { Aspergillus sojae } \\
\text { (domesticated from } \\
\text { A. parasiticus) }\end{array}$ & + & - & - & - & + & - & - & - \\
\hline Aspergillus tamarii & + & - & - & $+I-$ & - & - & - & - \\
\hline Aspergillus toxicarius & + & + & + & - & + & - & - & + \\
\hline Petromyces albertensis & + & - & - & - & - & + & - & - \\
\hline Petromyces alliaceus & + & - & - & - & - & + & - & - \\
\hline
\end{tabular}

Fonte: Samson; Hong; Frisvad, 2006, adaptado.

\section{$\underline{\text { Características moleculares }}$}

Os métodos moleculares têm sido amplamente aplicados na identificação de um grande número de espécies de Aspergillus (BATISTA et al., 2008). Estes estão entre os fungos mais bem estudados geneticamente. Na verdade, os genomas de várias espécies de Aspergillus estão concluídos (FEDOROVA et al., 2008; GALAGAN et al., 2005; MACHIDA et al., 2005; NIERMAN et al., 2005; PAYNE et al., 2006; ROKAS et al., 2007) e sequências de inúmeras linhagens encontram-se disponíveis.

Em geral, as características moleculares fornecem um grande número de características variáveis para a taxonomia de fungos, as quais são geradas usando-se uma tecnologia amplamente disponível, com uma infraestrutura de bioinformática 
extremamente bem desenvolvida que permite a comunicação em todo o mundo e a comparação de resultados. Essa técnica garante que as características moleculares tenham um papel fundamental no reconhecimento da espécie fúngica (GEISER et al., 2007).

Nos últimos anos, ferramentas moleculares como RFLPs, RAPDs, AFLP, MLEE, sequências ribosomais RNA e sequências de gene de proteínas codificadoras são aplicadas a questões taxonômicas do gênero. Estudos de sequências DNA multilocos de algumas espécies anamórficas têm mostrado que os padrões de polimorfismo em genes diferentes são consistentes com recombinação nestas espécies assexuadas (GEISER; PITT; TAYLOR, 1998). No entanto, a variedade de genes e métodos empregados nas pesquisas de identificação e filogenia baseadas no DNA têm limitado a aplicabilidade dos resultados para além dos grupos específicos de estudo (STOECKLI, 2003). Taylor et al. (2000) afirmam que a análise filogenética de características variáveis do ácido nucléico atualmente é um método mais próximo do que os outros de reconhecer as espécies de acordo com sua evolução, mas esso é considerado verdadeiro somente se vários genes são analisados. Para a descrição da espécie e a inferência filogenética, Samson e Varga (2009) recomendam que pelo menos duas sequências de genes devem ser examinadas usando-se a abordagem de Sequências Multilocos (MLST).

A internet oferece um meio para a distribuição rápida de dados, mas nem todos os sites na web contêm dados de igual qualidade. O GenBank, por exemplo, contém sequências de qualidade questionável e prováveis erros na identificação de organismos (GEISER et al., 2007).

Entretanto, mesmo com o alto nível de sensibilidade e resolução que os métodos moleculares normalmente mostram, alguns problemas ainda surgem no âmbito da seção Flavi, por causa do alto nível de similaridade genética entre algumas espécies (RODRIGUES et al., 2011).

Análise Proteômica - MALDI-TOF ICMS 
A espectrometria de massas pela técnica de MALDI-TOF MS surgiu no final de 1980 como uma técnica para investigar a espectrometria de massa de compostos orgânicos de elevada massa molecular através de uma ionização suave de moléculas, resultando em fragmentação mínima (TANAKA et al., 1988). Atualmente, este método tem sido utilizado para analisar a composição proteica de misturas complexas, isto é, fluidos biológicos ou tecidos, bem como de células microbianas ou componentes celulares (FENSELAU; DEMIREV, 2001; MAIER; KOSTRZEWA, 2007).

O funcionamento dessa técnica para células microbianas, de modo geral, ocorre da seguinte forma: a amostra contendo a espécie de interesse é misturada com uma matriz (geralmente um ácido orgânico aromático), formando uma "mistura sólida". Um pulso de laser, com comprimento de onda próximo do UV, incide sobre essa mistura, e a energia do laser é absorvida pela matriz, que tem o máximo de absorção perto do comprimento de onda do laser. Desse modo, a matriz evapora e o analito que estava incluso nela, passa para a fase gasosa, altamente energética, atribuída à excitação eletrônica da molécula da matriz ao absorver a energia do laser. A formação dos íons (ionização) ocorre através da transferência de carga (ex: transferência de prótons) das moléculas da matriz para o composto que fica na forma de $\mathrm{MH}+$. Os íons formados recebem uma alta energia cinética inicial $(\mathrm{K})$ que os impulsiona para o analisador de massas Time-Of-Flight (TOF), onde são separados de acordo com o tempo de voo, considerando a distância na qual o íon se movimenta até atingir o detector. Utilizando a equação da energia $\mathrm{K}=\mathrm{mV} 2 / 2$, onde $\mathrm{V}$ é a velocidade do íon, calcula-se a massa molecular do composto. Os compostos são separados no analisador de acordo com sua razão $\mathrm{m} / z$. Íons mais leves chegam mais rapidamente no detector, enquanto os íons mais pesados demoram mais tempo. No MALDI, o analisador de massas pode operar em dois modos: modo linear e modo reflectron. O modo linear é utilizado para moléculas de grande massa molecular, como as proteínas, os peptídeos e polímeros, e o modo reflectron é utilizado para moléculas de massa de até 7000Da, como as micotoxinas. O modo reflectron possui a vantagem de apresentar uma maior resolução quando comparado ao modo linear, devido à presença de um conjunto de lentes com diferença 
de potencial crescente, propiciando o aumento de resolução. Além disso, programas online estão disponíveis para permitir a detecção do conjunto massas/matriz, o que pode interferir nos resultados das amostras (KELLER et al., 2008; MARVIN; ROBERTS; FAY, 2003). Caracterização mais detalhada da técnica é relatada em um estudo realizado por Santos et al. (2010).

As vantagens de MALDI-TOF MS sobre as outras metodologias de espectrometria de massa são a simplificação da análise de massa espectral devida à ionização suave, reduzindo o número de sinais, tendo a faixa de massa mais ampla (KEMPTNER et al., 2009). Como resultado, muitas amostras complexas, como células inteiras, podem ser investigadas. Além disso, é uma técnica rápida e reprodutível, que tem sido utilizada com sucesso para a identificação e discriminação de vários microorganismos e tem mostrado altas potencialidades para discriminar taxon relacionados muito próximos.

Vários estudos têm sido realizados para o uso desse método. Santos et al. (2010) apresentam, em sua pesquisa de revisão, o uso do MALDI-TOF ICMS para a caracterização de fungos filamentosos. Sulc et al. (2008) utilizaram a abordagem da espectrometria de massas (MALDI-TOF) para a análise direta do esporo intacto de fungos do gênero Aspergillus e verificaram que a técnica tem a capacidade de diferenciar espécies dentro do mesmo gênero. Trabalho semelhante feito por Hettick et al. (2008) usou a técnica do MALD-TOF para gerar fingerprints de massa espectral reprodutível para 12 espécies de fungos do gênero Aspergillus e 5 diferentes cepas de Aspergillus flavus.

Kallow et al. (2006) relatam que, em fungos filamentosos, a maior parte dos sinais correspondem a proteínas ribossomais, cujas massas altamente características podem ser usadas para identificação e classificação. $\mathrm{Na}$ verdade, a composição da parede celular de fungos e também de esporos de fungos apresenta diferenças qualitativas e quantitativas em diferentes espécies de fungos, mas também entre as diferentes estirpes da mesma espécie fúngica (KEMPTNER et al., 2009). 
Autores atribuem as diferenças entre os fingerprints dos espectros de massa a vários fatores: instrumentação, nomeadamente, condições de cultivo e preparação da amostra e da matriz MALDI. Essas diferenças refletem a importância do uso da metodologia padronizada para o MALDI-TOF. Santos et al. (2010) enfatizam que uma biblioteca de referência padrão de espectros de massa biológica precisa ser implementada com base em testes interlaboratoriais.

\section{CONCLUSÃO}

O uso da abordagem polifásica, incluindo características fenotípicas, perfil micotoxigênico e análise molecular e espectral por MALDI-TOF MS faz-se necessário e indispensável para a caracterização de uma espécie. Além disso, é importante ressaltar que nenhuma das análises realizadas pode ser super ou subestimada, e a identificação de uma espécie de Aspergillus seção Flavi deve considerar todas as características analisadas.

\section{AGRADECIMENTOS}

À FAPEMIG - Fundação de Amparo à Pesquisa do Estado de Minas Gerais (Brasil) -, pelo apoio financeiro. À Capes - Coordenação de Aperfeiçoamento de Pessoal de Nível Superior (Brasil) -, pela bolsa de doutorado a F. C. Da Silva e pelo apoio financeiro a C. Santos e N. Lima como Professores Internacionais Visitantes no Programa de Pós-Graduação em Microbiologia Agrícola da Universidade Federal de Lavras, Lavras (MG), Brasil.

\section{REFERÊNCIAS}


BARROS, G. G. et al. Molecular characterization of Aspergillus section Flavi isolates collected from peanut fields in Argentina using AFLPs. Journal of Applied Microbiology, Oxford, v. 103, n. 4, p. 900-909, 2007.

BATISTA, P. P. et al. Genetic characterization of Brazilian strains of Aspergillus flavus using DNA markers. Genetics and Molecular Research, Ribeirão Preto, v. 7, n. 3, p. 706-717, 2008.

BENNETT, J. W.; KLICH, M. Mycotoxins. Clinical Microbiology Reviews, Washington, v. 16, n. 3, p. 497-516, June 2003.

CHANG, P. K.; EHRLICH, K. C.; HUA, S. S. T. Cladal relatedness among Aspergillus oryzae isolates and Aspergillus flavus $\mathrm{S}$ and $\mathrm{L}$ morphotype isolates. International Journal of Food Microbiology, Amsterdam, v. 108, n. 2, p. 172-177, June 2006.

COTTY, P. J. Virulence and cultural characteristic of two Aspergillus flavus strains pathogenic on cotton. Phytopathology, Saint Paul, v. 79, n. 7, p. 808-814, Sept. 1989.

EGEL, R. Mapping of additional markers in fission yeast, especially fus 1 and three mfm genes. Current Genetics, New York, v. 26, n. 2, p. 187-189, Aug. 1994.

FEDOROVA, N. D. et al. Genomic islands in the pathogenic filamentous fungus Aspergillus fumigatus. PLoS Genetics, San Francisco, v. 4, n. 4, p. 146-151, 2008.

FENSELAU, C.; DEMIREV, P. A. Characterization of intact microorganisms by maldi mass spectrometry. Mass Spectromety Reviews, New York, v. 20, n. 4, p. 157-171, 2001.

FRISVAD, J. C.; SKOUBOE, P.; SAMSON, R. A. Taxonomic comparison of three different groups of aflatoxin producers and a new efficient producer of aflatoxin B1, sterigmatocystin and 3-Omethylsterigmatocystin, Aspergillus rambellii sp. nov. Systematic and Applied Microbiology, Stuttgart, v. 28, n. 5, p. 442-453, May 2005.

GALAGAN, J. E. et al. Genomics of the fungal kingdom: insights into eukaryotic biology. Genome Research, Cold Spring Harbor, v. 15, n. 12, p. 1620-1631, Dec. 2005.

GEISER, D. M.; DORNER, J. W.; HORN, B. W.; TAYLOR, J. W. The phylogenetics of mycotoxin and sclerotium production in Aspergillus flavus and Aspergillus oryzae. Fungal Genetics and Biology, Orlando, v. 31, n. 3, p. 169-179, Mar. 2000.

GEISER, D. M.; PITT, J. I.; TAYLOR, J. W. Cryptic speciation and recombination in the aflatoxin-producing fungus Aspergillus flavus. Proceedings of the National 
Academy of Sciences of United States of America, Washington, v. 95, n. 1, p. 388393, 1998.

GODET, M.; MUNAUT, F. Molecular strategy for identification in Aspergillus section Flavi. FEMS Microbiology Letters, Amsterdam, v. 304, n. 2, p. 157-168, Feb. 2010.

GONÇALVES, S. S. et al. Aspergillus novoparasiticus: a new clinical species of the section Flavi. Medical Mycology, Oxford, v. 50, n. 4, p. 152-160, 2012.

GOTO, T.; WICKLOW, D. T.; ITO, Y. Aflatoxin and cyclopiazonic acid production by a Sclerotium-producing Aspergillus tamarii strain. Applied and Environmental Microbiology, Washington, v. 62, n. 11, p. 4036-4038, Nov. 1996.

HONG, S. B. et al. Polyphasic taxonomy of Aspergillus fumigatus and related species. Mycologia, New York, v. 97, n. 6, p. 1316-1329, 2005.

HORN, B. W. Aspergillus caelatus, a new species in section Flavi. Mycotaxon, Ithaca, v. 56, n. 2, p. 185-191, 1997.

HORN, B. W. et al. Association of morphology and mycotoxin production with vegetative compatibility groups in Aspergillus flavus, A. parasiticus, and A. tamarii. Mycologia, New York, v. 88, n. 4, p. 574-587, Aug. 1996.

HEDAYATI, M. T. et al. Aspergillus flavus: human pathogen, allergen and mycotoxin producer. Microbiology, New York, v. 153, n. 6, p. 1677-1692, June 2007.

HETTICK, J. M. et al. Discrimination of Aspergillus isolates at the species and strain level by matrix-assisted laser desorption/ionization time-of-flight mass spectrometry fingerprinting. Analytical Biochemistry, New York, v. 380, n. 2, p. 276-281, Feb. 2008.

HUSSEIN, H. S.; BRASEL, J. M. Toxicity, metabolism and impact of mycotoxins on humans and animals. Toxicology, Limerick, v. 167, n. 2, p. 101-134, Feb. 2001.

ITO, Y. et al. Aspergillus pseudotamarii, a new aflatoxin producing species in Aspergillus section Flavi. Mycological Research, Cambridge, v. 105, n. 2, p. 233-239, Apr. 2001.

JOHNSSON, P. et al. Growth of aflatoxigenic moulds and aflatoxin formation in Brazil nuts. World Mycotoxin Journal, Wageningen, v. 1, n. 2, p. 127-137, June 2008.

KALLOW, W. et al. Microbial strain characterisation by MALDI-TOF MS possibilities and limits. In: ANNUAL GENERAL MEETING OF THE EUROPEAN CULTURE 
COllections' ORGAniSATION, 10., 2006, Budapest. Proceedings... Budapest: Corvinus University of Budapest, 2006. p. 146-159.

KELLER, B. O. et al. Interferences and contaminants encountered in modern mass spectrometry. Analytica Chimica Acta, Amsterdam, v. 627, n. 1, p. 71-81, Mar. 2008.

KEMPTNER, J. et al. Evaluation of matrix-assisted laser desorption/ionization (MALDI) preparation techniques for surface characterization of intact Fusarium spores by MALDI linear time-of-flight mass spectrometry. Rapid Communications in Mass Spectrometry, Chichester, v. 23 , n. 6 , p. $877-884$, June 2009.

$\mathrm{KLICH}$, M. A. Environmental and developmental factors influencing aflatoxin production by Aspergillus flavus and Aspergillus parasiticus. Mycoscience, Tokyo, v. 48, n. 4, p. 1-80, 2007.

KLICH, M. A.; PITT, J. L. Differentiation of Aspergilus flavus and A. parasiticus and other closely related species. Transactions of the British Mycological Society, Cambridge, v. 91, n. 1, p. 99-108, Apr. 1988.

KOZAKIEWICZ, Z. Aspergillus species on stored products. Mycological Papers, Geneva, n. 161, p. 1-188, 1989.

KUMEDA, Y.; ASAO, T. Heteroduplex panel analysis, a novel method for genetic identification of Aspergillus section Flavi strains. Applied and Environmental Microbiology, Washington, v. 67, n. 9, p. 4084-4090, Sept. 2001.

KURTZMAN, C. P.; HORN, B. W.; HESSELTINE, C. W. Aspergillus nomius, a new aflatoxin-producing species related to Aspergillus flavus and Aspergillus tamari. Antonie van Leeuwenhoek, Amsterdam, v. 53, n. 3, p. 147-158, 1987.

LARSEN, T. et al. Phenotypic taxonomy and metabolite profiling in microbial drug discovery. Natural Product Reports, London, v. 22, n. 6, p. 675-695, Dec. 2005.

LI, D. M. et al. Three new Aspergillus species isolated from clinical sources as a causal agent of human aspergillosis. Mycoscience, Tokyo, v. 39, n. 3, p. 299-305, June 1998.

MACHIDA, M. et al. Genome sequencing and analysis of Aspergillus oryzae. Nature, London, v. 438, n. 7071, p. 1157-1161, 2005.

MARVIN, L. F.; ROBERTS, M. A.; FAY, L. B. Matrix-assisted laser desorption/ionization time-of-flight mass spectrometry in clinical chemistry. Clinica Chimica Acta, Amsterdam, v. 337, n. 4, p. 11-21, Apr. 2003. 
NIERMAN, W. C. et al. Genomic sequence of the pathogenic and allergenic filamentous fungus Aspergillus fumigatus. Nature, London, v. 438, n. 7071, p. 11511156, 2005.

PATERSON, R. R. M. Fungal enzyme inhibitors as pharmaceuticals, toxins, and scourge of PCR. Current Enzyme Inhibition, Hilversum, v. 4, n. 1, p. 46-59, Mar. 2008 .

PAYNE, G. A. et al. Whole genome comparison of Aspergillus flavus and A. oryzae. Medical Mycology, Oxford, v. 44, n. 1, p. 9-11, Feb. 2006.

PETERSON, S. W. Phylogenetic analysis of Aspergillus species using DNA sequences from four loci. Mycologia, New York, v. 100, n. 2, p. 205-226, Feb. 2008.

PETERSON, S. W. et al. Aspergillus bombycis, a new aflatoxigenic species and genetic variation in its sibling species, A. nomius. Mycologia, New York, v. 93, n. 4, p. 689-703, Apr. 2001.

PILDAIN, M. B. et al. Two novel aflatoxin-producing Aspergillus species from Argentinean peanuts. International Journal of Systematic and Evolutionary Microbiology, Reading, v. 58, n. 3, p. 725-735, June 2008.

OLSEN, M. et al. Aspergillus nomius, an important aflatoxin producer in Brazil nuts? World Mycotoxin Journal, Wageningen, v. 1, n. 2, p. 123-126, 2008.

RAPER, K. B.; FENNEL, D. I. The genus Aspergillus. Baltimore: Williams \& Wilkins, $1965.686 \mathrm{p}$.

RIGÓ, K. et al. Evolutionary relationships within Aspergillus section Flavi based on sequences of the intergenic transcribed spacer regions and the 5.8S rRNA gene. Journal of General Applied Microbiology, Tokyo, v. 48, n. 1, p. 9-16, Jan. 2002.

RODRIGUES, P. et al. Species identification of Aspergillus section Flavi isolates from Portuguese almonds using phenotypic, including MALDI-TOF ICMS, and molecular approaches. Journal of Applied Microbiology, Oxford, v. 111, n. 4, p. 877-892, Aug. 2011.

RODRIGUES, P. et al. A polyphasic approach to the identification of aflatoxigenic and non-aflatoxigenic strains of Aspergillus section Flavi isolated from Portuguese almonds. International Journal of Food Microbiology, Amsterdam, v. 129, n. 2, p. 187-193, June 2009. 
ROKAS, A. et al. What can comparative genomics tell us about species concepts in the genus Aspergillus? Studies in Mycology, Berlin, v. 59, n. 1, p. 11-17, Jan. 2007.

SAITO, M.; TSURUTA, O. A new variety of Aspergillus flavus from tropical soil in Thailand and its aflatoxins productivity. Proceedings of the Japanese Association of Mycotoxicology, Tokyo, v. 37, n. 1, p. 31-36, 1993.

SAMSON, R. A.; VARGA, J. Molecular systematics of Aspergillus and its teleomorphs. In: MASHIDA, M.; GOMI, K. (Ed.). Aspergillus: molecular biology and genomics. London: Caister Academic, 2010. p. 19-40.

SAMSON, R. A.; VARGA, J. What is a species in Aspergillus? Medical Mycology, Oxford, v. 47, n. 1, p. 13-20, Jan. 2009.

SAMSON, R. A.; VARGA, J. Polyphasic taxonomy of Aspergillus section Fumigati and its teleomoorph Neosartorya. Studies in Mycology, Berlin, v. 59, n. 1, p. 147-207, Jan. 2007.

SAMSON, R. A.; HONG, S. B.; FRISVAD, J. C. Old and new concepts of species differentiation in Aspergillus. Medical Mycology, Oxford, v. 44, n. 1, p. S133-S148, 2006. Supplement.

SAMSON, R. A.; HONG, S. B.; FRISVAD, J. C. Methods for the detection, isolation and characterisation of food-borne fungi. In: SAMSON, R. A.; HOEKSTRA, E. S.; FRISVAD, J. C. (Ed.). Introduction to food and airborne fungi. $7^{\text {th }}$ ed. Wageningen: CBS, 2004. p. 283-305.

SAMSON, R. A. et al. Identification of the common food and airborne fungi, Aspergillus. In: SAMSON, R. A. et al. Introduction to food and airborne fungi. Utrecht: Centraalbureau voor Schimmekultures, 2000. p. 64-97.

SANTOS, C. et al. Filamentous fungal characterizations by matrix-assisted laser desorption /ionization time-of-flight mass spectrometry. Journal of Applied Microbiology, Oxford, v. 108, n. 2, p. 375-385, Feb. 2010.

SOARES, C. et al. Three new species of Aspergillus section Flavi isolated from almonds and maize in Portugal. Mycologia, New York, 2011. In press.

STOECKLI, E. RNAi in avian embryos. In: HANNON, G. J. (Ed.). RNA: a guide to gene silencing. Cold Spring Harbor: Cold Spring Harbor Laboratory, 2003. p. 297-312. 
SULC, M. et al. Biomarkers of Aspergillus spores: strain typing and protein identification. International Journal of Mass Spectrometry, London, v. 280, n. 1/3, p. 162-168, 2008.

TANAKA, K. et al. Protein and polymer analyses up to m/z 100000 by laser ionization time-of-flight mass spectrometry. Rapid Communications in Mass Spectrometry, Chichester, v. 2, n. 8, p. 151-153, Aug. 1988.

TAYLOR, J. W. et al. Phylogenetic species recognition and species concepts in fungi. Fungal Genetics and Biology, Orlando, v. 31, n. 1, p. 21-32, Feb. 2000.

VARGA, J. et al. Evolutionary relationships among Aspergillus species producing economically important mycotoxins. Food Technology and Biotechnology, Zagreg, v. 41, n. 1, p. 29-36, Jan. 2003.

YU, J. et al. Aspergillus flavus genomics: gateway to human and animal health, food safety, and crop resistance to diseases. Revista Iberoamericana de Micologia, Barcelona, v. 22, n. 4, p. 194-202, 2005. 